\title{
Congenital convex concha-An unfamiliar auricular deformation and a novel non-surgical management protocol
}

\author{
Asaf Olshinka ${ }^{1,2 *}$, Dafna Yaacobi ${ }^{1}$ and Dean Ad-El ${ }^{1}$ \\ ${ }^{1}$ Department of Plastic Surgery and Burns, Rabin Medical Center, 39 Zabutinsky st, Petah Tikva 4941492 Israel \\ ${ }^{2}$ Plastic Surgery and Burns Unit, Schneider Children's Medical Center of Israel, 14 Kaplan Street, Petah Tikva 4920235, Israel
}

\begin{abstract}
Objective: Ear anomalies are observed in approximately 30\% of new-borns of which 15-20\% do not resolve spontaneously. Whereas malformations can be amended only surgically, deformations, if diagnosed early, are correctable non-surgically, with molding/splinting techniques. The convex- concha deformity is an unfamiliar deformation, which is not only aesthetic but may also interrupt with hearing and usage of hearing-aids. The aim of the present report was to describe the deformity and the use of the novel non-invasive treatment protocol for its management.
\end{abstract}

Subjects and methods: The cohort included 10 new-borns treated for congenital convex concha in 2018-2019. All underwent conservative management using the EarWell ${ }^{\circledR}$ system, a device consisting of four interlocking components that reshape and reposition the ear. In some cases, the system was combined with molding and taping for optimal results.

Results: All patients began treatment before age 3 weeks (average, 2 weeks). The average duration of treatment was 5.2 weeks. $90 \%$ showed no complications. Most of parents were pleased with the aesthetic outcome; $70 \%$ were extremely satisfied.

Conclusions: This study showed that early use of our treatment-protocol for new-borns with a convex-concha deformation led to good results within a relatively short period without the need for invasive procedures; Our cohort demonstrated that an earlier onset of treatment results in a shorter treatment-period.

\section{Introduction}

Congenital ear anomalies are very common. Approximately $30 \%$ of new-borns have some degree of auricle anomaly [1], of which $15-20 \%$ resolve spontaneously [2]. Anomalies are divided into malformation and deformations. While deformation is characterized by fully developed auricular components (full pinna) and can be manually corrected into normal shape; malformations are due to abnormal embryologic development of the auricle and may affect the inner or outer ear (missing or excess anatomic structures, otherwise anomalous pinna) [2]. Common malformations include constriction, anotia, cleft ear, and microtia [3]; all require surgical correction. Deformations are characterized by completely developed auricular components (full pinna) and include anomalies such as prominent ear, helical rim anomalies, Stahl ear, lidding/lop ear, conchal strut/crus, and cryptotia [4]. If diagnosed early, the majority of ear deformations can be treated conservatively $[3,5]$ by molding or splinting, with satisfactory results [6].

Conservative treatment for ear deformations were first described in 1980 [7]. Since then, many types of techniques have been reported, mostly involving the use of foam, surgical tapes, wires, and other materials [6,8]. All are malleable, light, and non-irritating [9].

The authors use different, case-specific techniques for nonsurgical treatment. One of the main tools is the EarWell ${ }^{\bullet}$ Infant Ear Correction System (Becon Medical, Naperville, IL, USA), which has been proven to achieve excellent results ${ }^{6,10}$ in managing deformations and even reducing the severity of some malformations [2]. The system consists of four interlocking components: a large posterior shell that fits around the ear and is held to the scalp with adhesive tape; a conchal former that shapes the conchal bowl of the ear; small soft rubber retractors that gently shape the ear to the desired position; and a snap-on outer lid of soft breathable rubber that holds the components securely in place.

Congenital convex concha is one of the less common cartilaginous auricular framework deformities [11], especially as an isolated anomaly [6] (Figure 1). It warrants particular attention because besides its adverse aesthetic effects, it can also interrupt hearing and prevent the use of a hearing aid. In severe cases, patients might present with stenosis of the auditory canal meatus which may lead to hearing loss $[12,13]$. Surgical treatments have been described for this condition [14-16], but only one case report described a conservative approach consisting of splinting for 10 weeks, initialed at the age of 3 months [13].The aim of the present report was to describe the convex concha deformity and its management by a novel noninvasive treatment protocol based on the EarWell $^{\circledR}$ system.

${ }^{*}$ Correspondence to: Asaf Olshinka, Plastic Surgery \& Burns Unit, Schneider Children's Medical Center of Israel, 14 Kaplan Street, Petah Tikva 4920235, Israel, Tel: +972-3-9376370; Fax: +972-3-9219773; E-mail: asafol@clalit.org.il

Key words: conchal deformation, concave concha, nonsurgical treatment, ear molding, ear splinting, cartilage maleability

Received: February 11, 2020; Accepted: February 25, 2020; Published: February 27, 2020 


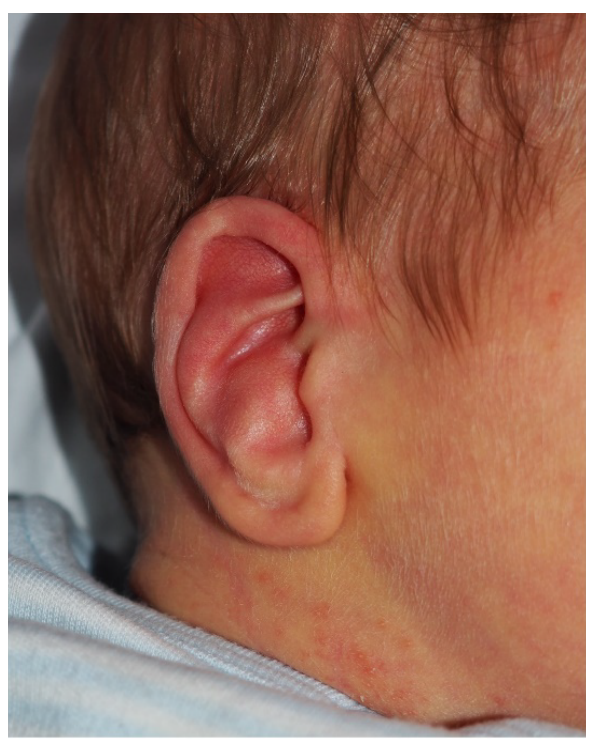

Figure 1. Patient with a convex concha deformation of the right ear

\section{Materials and methods}

\section{Patients and setting}

The cohort consisted of 10 new-borns who presented with congenital convex concha at our clinic in 2018-2019. All underwent treatment with the EarWell ${ }^{\circledR}$ system. The study was approved by the local institutional review board. Parents provided written consent for use of the EarWell ${ }^{\circ}$ system.

\section{The EarWell ${ }^{\circledR}$ system}

Treatment with the EarWell ${ }^{\circledR}$ system was started as early as possible, preferably in the first days of life and at least during the first two weeks. The size selected (medium or large) was based on the size of the auricle. The former was used to reshape the concha by applying gentle downward pressure on the conchal cartilage. A custom-made silicone ear mold (Azoft; Detax, Ettlingen, Germany) was added later for contouring depending on the rate and extent of improvement. Additional anomalies were treated with the appropriate retractors (Figure 2).

\section{Follow-up protocol}

Patients are followed for conchal concavity once weekly in the clinic. According to our protocol, wounds or ulcers were to be treated with antibiotic ointment and a local dressing. In cases of significant ulcers, the protocol would be stopped (Figure 3).

Treatment with the EarWell ${ }^{\circledR}$ system was continued until an appropriate conchal concavity was reached. When the initial response was rapid (improvement within one week), we discontinued use of the EarWell ${ }^{\circ}$ after 2 weeks of treatment and switched to posterior taping for an additional 2 weeks. When improvement was delayed beyond 2 weeks of treatment, treatment was continued for an additional 2 weeks after which the EarWell ${ }^{\circ}$ was replaced with silicone molding (Figure 4) for 1-2 weeks (Figure 5) followed by posterior taping for an additional 2 weeks.

\section{Results}

The cohort included male and female patients with a convex concha deformation and had an additional anomaly. All were treated with the EarWell ${ }^{\triangleright}$ system within the first 3 weeks of life (Figure 6); the average patient age at treatment initiation was 2 weeks (Figure 6). Average duration of treatment was 5.2 weeks. $10 \%$ had ulceration and dermatitis and $30 \%$ had isolated dermatitis (Table 1). This process resulted in an aesthetic and natural conchal appearance (Figure 7). Most of the parents were satisfied with the results, and $70 \%$ were extremely satisfied (Figure 8).

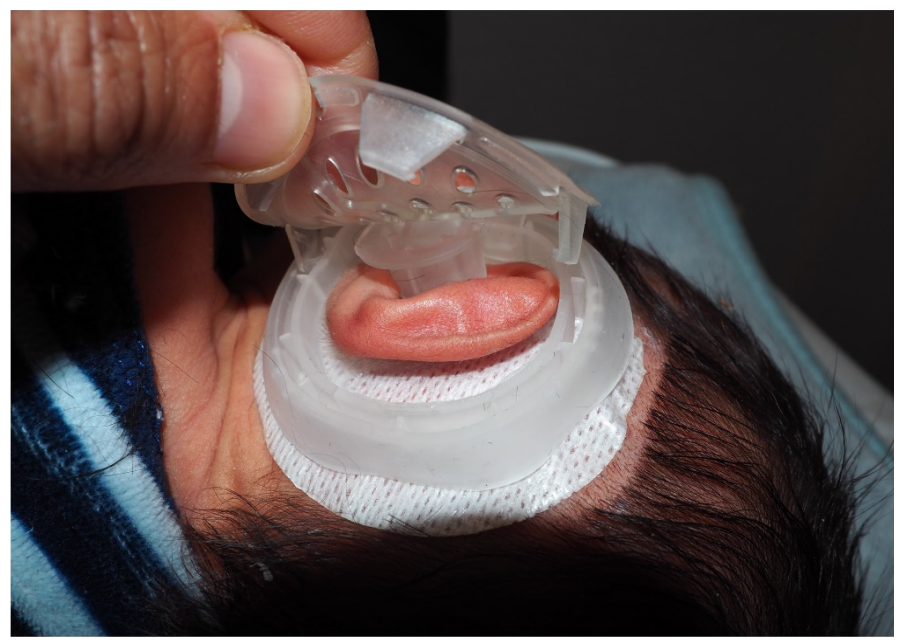

Figure 2. Conchal former (EarWell ${ }^{\circledR}$ system) applied to the left ear of a patient
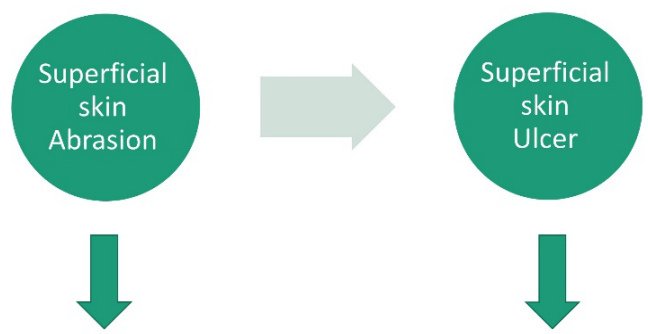

- Antibiotic ointment

- Local dressing

- Continue treatment

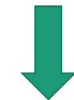

- STOP treatment

- Treat wound

Figure 3. Adverse effects, treatment protocol

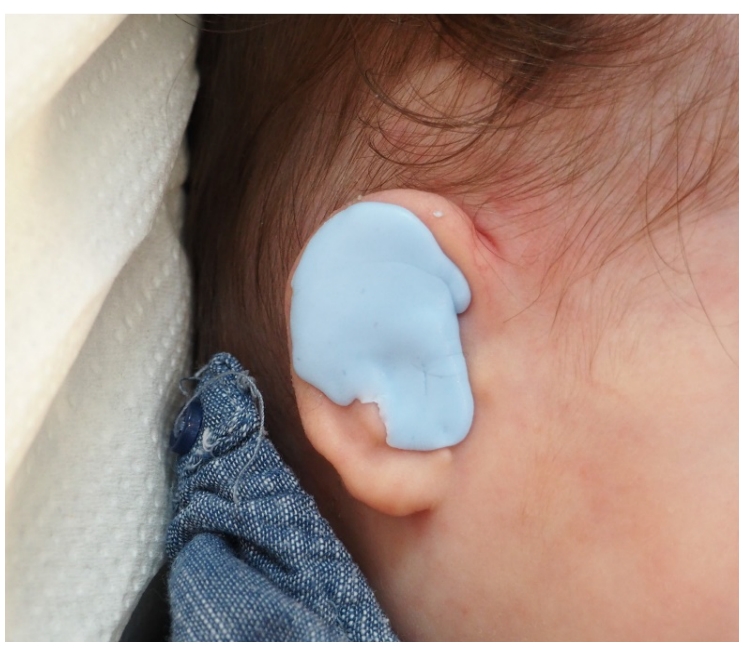

Figure 4. Silicone mold applied at the second stage on the right ear 


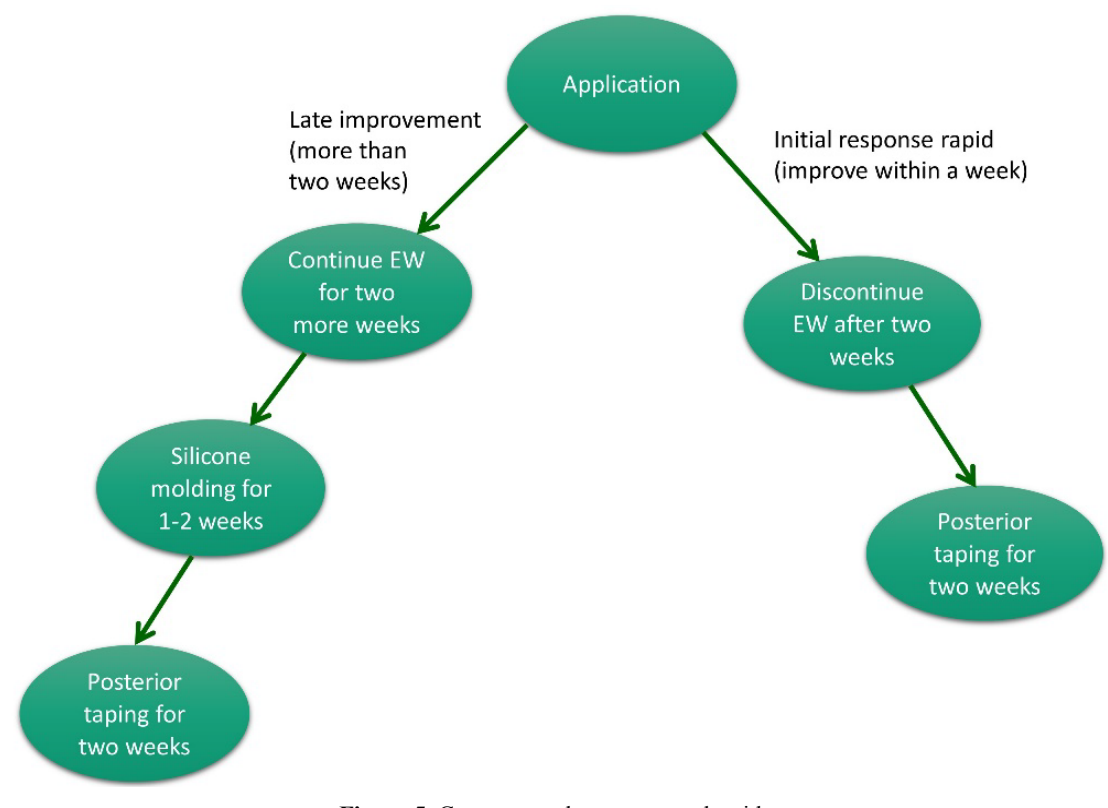

Figure 5. Convex concha treatment algorithm

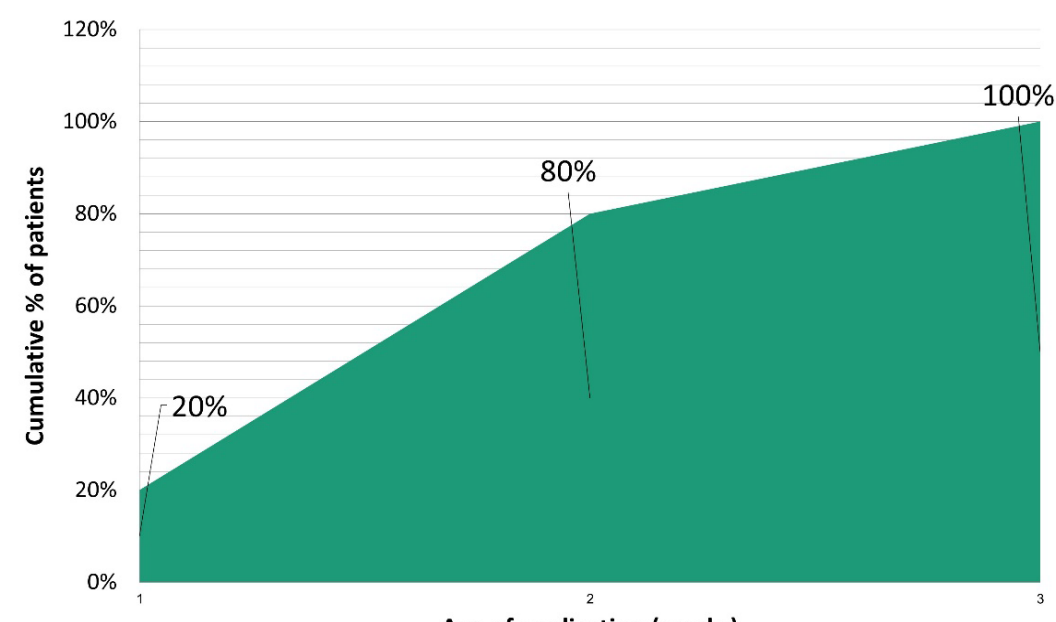

Age of application (weeks)

Figure 6. Right ear before and after treatment of a convex concha deformation

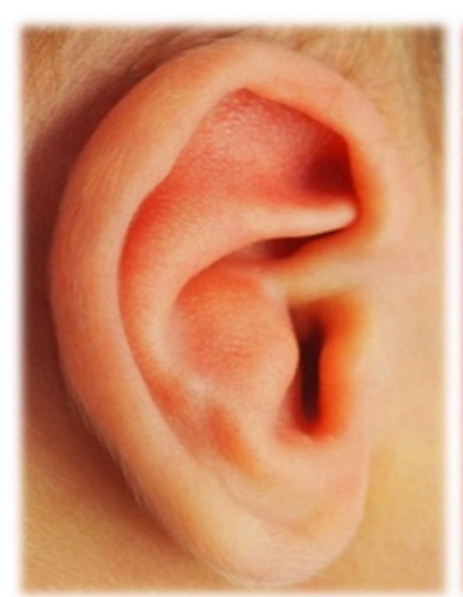

Before



After

Figure 7. Average age (in weeks) of application (cumulative) 


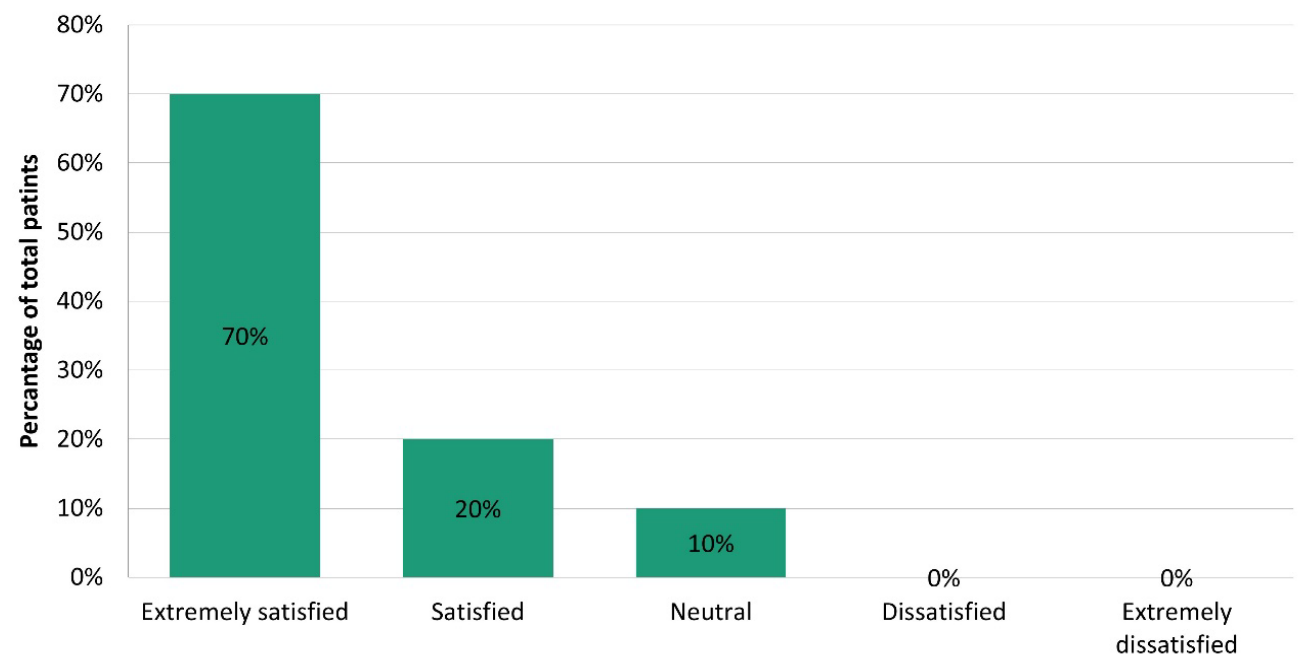

Parents satisfaction rate

Figure 8. Parental satisfaction rates

7



0

Age of application (weeks)

Figure 9. Mean duration of treatment by age (in weeks) at application

\section{Discussion and conclusions}

Given that congenital auricular deformities are common and only 15-20\% resolve spontaneously [6], it is important that pediatricians and health care providers be able to identify and explain the condition to the parents right after birth. The cases need to be referred for treatment as soon as possible in order to spare patients unnecessary invasive procedures and ease parental anxiety [10]. Although some authors claim conservative means may be applied up to age 3 months [17], it is known that estrogen is responsible for the malleability and plasticity of the auricular cartilage through increased levels of hyaluronic acid, and that hyaluronic acid levels peak within 72 hours from birth, followed a gradual decrease to baseline 4 days later. Therefore, the ideal timing for manual treatment of auricular deformities is the first 2 weeks of life. The more treatment is delayed, the longer the treatment period necessary and the lower the success rate [10].
Most auricular anomalies are located in the helical rim, [2,4] and clinicians have greater knowledge and experience treating them compared to anomalies located closer to the auditory canal. Among the conchal anomalies, the most abundantly described is conchal crus malformation, which is characterized by an abnormal fold of cartilage that crosses through the center of the conchal bowl. To our opinion, this should be categorized as an anatomical variant rather than an anomaly because it has only aesthetic consequences. In contrast, convex concha deformation, besides its aesthetic repercussions, may also result in functional impairments due to auditory canal stenosis and interfere with the use of hearing aids owing to its location $[12,13]$. Therefore, it is important that it be addressed particular attention. We present an unfamiliar auricular deformation and our non-surgical treatment protocol for it. In our experience the combined treatment of convex concha combining the EarWell ${ }^{\circ}$ system with a silicone mold and taping yields favorable and consistent results. It is not unusual for convex concha to present with other ear deformities. These can be corrected simultaneously with the EarWell ${ }^{\circ}$ system. 
In agreement with the literature, we found that when treatment was started early, a relatively short treatment period was required to achieve the desired result (Figure 9). We assume this finding is attributable to the decreased malleability of the cartilage with age owing to a decline in estrogen levels [10,17].

Like for other types of auricular deformations, if non-surgical treatment of convex concha cannot be performed in the appropriate window of time, surgical intervention is the next best option. This may include partial excision of the conchal cartilage and closure with permanent stiches. The timing of surgery depends on patient maturity and ear development. In the majority of cases, it is not be performed earlier than age 6 years, by which time there has been significant relative growth of the ear [18]. Surgery is associated with higher rates of complications and recurrence than conservative treatment and higher costs [19].

\section{References}

1. Byrd HS, Langevin CJ, Ghidoni LA (2010) Ear molding in newborn infants with auricular deformities. Plast Reconstr Surg 126: 1191-1200. [Crossref]

2. Daniali LN, Rezzadeh K, Shell C, Travato M, Ha R, et al. (2017) Classification of newborn ear malformations and their treatment with the EarWell Infant Ear Correction Systemi. Plast Reconstr Surg 139: 681-691. [Crossref]

3. Doft M, Goodkind A, Diamond S, DiPace JI, Kacker A, et al. (2015) The newborn butterfly project: a shortened treatment protocol for ear molding. Plast Reconstr Surg 135: 577e-583e. [Crossref]

4. Chan SLS, Lim GJS, Por YC, Chiang MF, Ho S, et al. (2019) Efficacy of ear molding in infants using the EarWell Infant Correction System and factors affecting outcomet. Plast Reconstr Surg 144: 648e-658e. [Crossref]

5. Kurozumi N, Ono S, Ishida H (1982) Non-surgical correction of a congenital lop ear deformity by splinting with Reston foaml. Br J Plast Surg 35: 181-182. [Crossref]

6. Schönauer F, Martino A Di, Gault DT (2015) Anteverted concha: a new ear deformational anomaly'. JPRAS Open 5: 46-50.
7. Tan ST, Abramson DL, MacDonald DM, Mulliken JB (1997) Molding therapy for infants with deformational auricular anomalies. Ann Plast Surg 38: 263-268. [Crossref]

8. Anstadt EE, Johns DN, Kwok AC-M, Siddiqi F, Gociman B (2016) Neonatal ear molding: timing and techniqué. Pediatrics 137: e20152831.

9. Leonardi A, Bianca C, Basile E, Ungari C, Arangio P, et al. (2012) Neonatal molding in deformational auricular anomalies. Eur Rev Med Pharmacol Sci 16: 1554-1558. [Crossref]

10. Matsuo K, Hirose T, Tomono T, Iwasawa M, Katohda S, et al. (1984) Nonsurgical correction of congenital auricular deformities in the early neonate: a preliminary report. Plast Reconstr Surg 73: 38-51. [Crossref]

11. Furnas DW (1999) Nonsurgical treatment of auricular deformities in neonates and infantś. Pediatr Ann 28: 387-390. [Crossref]

12. Ullmann Y, Blazer S, Ramon Y, Blumenfeld I, Peled IJ (2002) Early nonsurgical correction of congenital auricular deformities. Plast Reconstr Surg 109: 907-913. [Crossref]

13. Hong P (2012) Reconstruction of congenital inverted conchal bowl deformity: Our experience in two of four children. Clin Otolaryngol 37: 409-411. [Crossref]

14. Baklacı D (2018) Inverted concha, a rare auricular deformity with literature review. Entcase.

15. Osmani OA, Matthews R (2016) An original technique for the correction of congenital inverted conchal bowl deformity in an adult. Eur J Plast Surg 39: 449-452.

16. Shetty SC, Gupta S, Hasan S, Cherian M, Joseph V, et al. (2000) Reverse (convex) conchal bowl: Report of eight cases and its correction by modified conchoplasty technique. Can J Plast Surg 8: 233-238.

17. Harounian J, Fundakowski C (2017) Reconstruction of the conchal bowl. Operat Tech Otolaryngol Head Neck Surg 28: 125-129.

18. Janz B A, Cole P, Hollier LH Jr, Stal S (2009) Treatment of prominent and constricted ear anomalies. Plast Reconstr Surg 124: 27e-37e.! [Crossref]

19. Adamson JE, Hortox C E, Crawford HH (1965) The growth pattern of the external ear Plast Reconstr Surg 36: 466-470.] [Crossref]

Copyright: (C2020 Olshinka A. This is an open-access article distributed under the terms of the Creative Commons Attribution License, which permits unrestricted use, distribution, and reproduction in any medium, provided the original author and source are credited. 